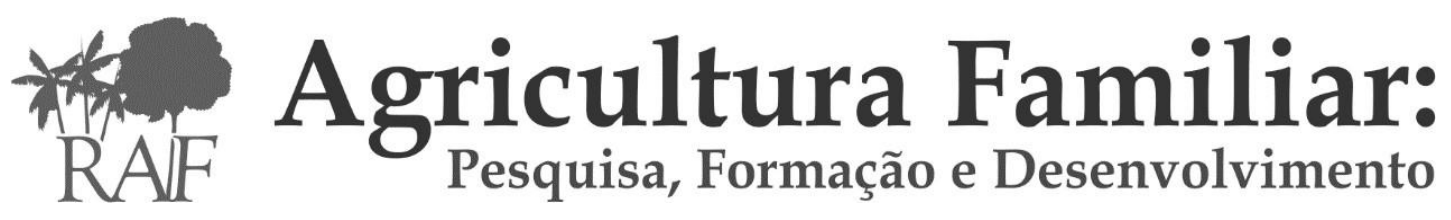

RAF. v.14 , nº 1 / jan-jun 2020, ISSN 1414-0810

\title{
Caracterização da pecuária leiteira de base familiar no Estado do Pará: reflexões sobre práticas agroecológicas
}

\section{Characterization of family dairy farming in the State of Pará: discussion on agroecological practices}

Laura Angélica Ferreira, Doutora, UFPA, laurange@ufpa.br;

Livio Sérgio Dias Claudino, Doutor, UFPA, livio.claudino@gmail.com;

Soraya Abreu de Carvalho, Doutora, UFPA, soraya@ufpa.br;

Rosana Quaresma Maneschy, Doutora, UFPA, romaneschy@ufpa.br;

René Poccard-Chapuis, Doutor, CIRAD, poccardchapuis@gmail.com;

\section{Resumo}

Assumindo a importância da produção leiteira na composição dos sistemas agrícolas familiares de base camponesa, assim como as controvérsias sociais e ambientais que a envolvem, este artigo busca refletir sobre a existência e a prática de sistemas agroecológicos nesta atividade no estado do Pará. A metodologia de trabalho utilizada foi a leitura e reflexão a partir de estudos desenvolvidos envolvendo diversas equipes de pesquisas, incluindo os autores deste artigo, que, ao longo de mais de 20 anos, têm estudado a pecuária leiteira na Amazônia paraense. Como principais resultados, identificamos que não há uma classificação exata para o sistema leiteiro de base familiar na Amazônia paraense, podendo-se dizer apenas que, de uma maneira geral, se trata de sistemas naturais e sem muitos incrementos, fruto da percepção e experiências empíricas dos atores sobre as necessidades de interação visando a produção dentro do novo ecossistema.

\section{Palavras-chave}

Amazônia; Agroecologia; Manejo; Pecuária bovina.

\section{Abstract}

Dairy production, integral to family farming systems, is essential to many peasant groups. However, many social and environmental controversies are associated with this activity. This article seeks to reflect on the existence and practice of agro-ecological dairy systems in Pará state. Our methods included a review and reflection on studies involving several research teams, including the authors of this article, who have researched dairy farming in the Amazon (Pará state) for over 20 years. Through our research we identified that no exact classification for the family-based dairy system in the Pará state exists. In general, they are represented by natural systems with few inputs and are resultant of actors' perceptions and empirical experiences based on interactions, aiming for production within the local ecosystem.

\section{Keywords}

Amazon; Agroecology; Animal Management; Cattle raising. 


\section{Introdução}

Pelo menos desde os anos 1990, o avanço da pecuária bovina na Amazônia foi apontado como grande causador de impactos nas dimensões ambiental, econômica e social, quando a atividade estava se tornando predominante entre agricultores familiares. Essa situação preocupava parte do meio acadêmico, já que a pecuária bovina extensiva demandaria grandes áreas, as quais não estavam disponíveis para a maior parte do segmento. Esse contexto fomentou reflexões sobre a inviabilidade técnica, social e ambiental da atividade, especialmente em áreas pequenas e localizadas nas fronteiras agrícolas, como era o caso do estado do Pará (FERREIRA, 2001).

A principal justificativa era a de que a agricultura familiar na Amazônia paraense se desenvolvia com práticas ditas extensivas, que ora representavam a melhor forma de manejo produtivo, em termos da relação custo/benefício e no ambiente em que se encontrava; ora se caracterizavam pela rusticidade e inadequação do uso do recurso natural manejado. Isso divergia de outras regiões do país, como no Rio Grande Sul, onde a pecuária foi apontada como uma atividade favorável à manutenção da biodiversidade, no caso, nos campos nativos (BORBA; TRINDADE, 2009).

Os trabalhos desenvolvidos, especialmente depois dos anos 2000, foram direcionados especialmente sobre o tema do avanço da pecuária bovina em áreas de floresta e na própria agricultura familiar no estado do Pará. Até então, a preocupação central era conhecer as dinâmicas, indicar cenários futuros e buscar meios para superar as principais limitações, sendo algumas delas: o acelerado processo de degradação das pastagens, a rápida migração das famílias para novas áreas e o consequente desmatamento para implantação de pastagens, aliados à baixa produtividade animal por área. Expressivo contingente de recursos econômicos e humanos foi investido à época para tais missões. Embora ainda muito predominante em algumas regiões do estado do Pará, aos poucos, em determinados casos, a pecuária foi perdendo espaço para outras atividades agrícolas, como o monocultivo de soja (Glycine max L. Merrill.) ou de dendê (Elaeis guineensis Jacq.) (SILVA, NAVEGANTES-ALVES, 2017).

Algumas questões foram sendo gradualmente respondidas, outras perderam a relevância, e novas questões foram surgindo. Uma delas, que é a inquietação proposta 
neste artigo, é sobre a viabilidade de se constituir, em nível estadual, uma pecuária bovina leiteira em estabelecimentos familiares fundamentada nos princípios da agroecologia, utilizando o perfil socioeconômico, cultural e técnico já existente.

De maneira mais conceitual, Gliessman (2001) define que a produção agroecológica implica em práticas no manejo do agroecossistema baseadas em princípios e conceitos da ecologia referente ao ecossistema em que está inserida. Altieri (2012) destaca que o manejo e a preservação dos recursos naturais devem estar em consonância com a cultura local, sendo socialmente justa e economicamente viável. Já Caporal e Costabeber (2004) nos indicam que a produção agroecológica não é um modelo ecológico de produção, mas uma forma de pensar a vida e, consequentemente, a agricultura com outros parâmetros que não os atuais econômicos e produtivos.

Balem e Machado (2019) demonstram que para o caso da pecuária leiteira, é primordial o papel da extensão rural com ênfase no manejo ecológico de solos; bem-estar animal; práticas de sanidade baseadas na homeopatia e fitoterapia; respeito à relação ser humano-natureza e a reprodução social, sendo que a produção dos conhecimentos deve levar em conta o saber dos atores locais.

No caso da pecuária leiteira, os modelos de produção mais difundidos são baseados em um alto consumo de insumos externos, relacionados à alimentação e aos medicamentos utilizados, principalmente para uma melhor adaptação ao clima tropical dos animais originalmente de climas temperados. Desta forma, sua evolução em termos de produtividade foi sempre baseada na artificialização do meio como forma de ultrapassar as barreiras genéticas e climáticas para altas performances. Falar de sistema agroecológico leva, num primeiro momento, à reflexão sobre a diminuição dos insumos utilizados e à reflexão da complexidade dos diversos sistemas em prática. Os estudos nesta área privilegiam tratar alguns elementos por vez e não o sistema como um todo. Como é o caso de estudos sobre manejo sanitário com homeopatia e fitoterápicos; sistema de integração a partir do manejo da fertilidade nos sistemas de cultivo; bem-estar animal. Mas o que se tem refletido sobre um sistema leiteiro agroecológico em sua globalidade?

Frente a essa contextualização, o presente texto tem por finalidade responder à questão: é possível falar de produção leiteira familiar de base agroecológica na Amazônia paraense? Para responder tal pergunta, apresentamos em seguida uma revisão da literatura sobre agroecologia e pecuária leiteira, indicando o estado da arte e, em seguida, a 
metodologia. No item 3, apresentamos os resultados, principalmente oriundos das pesquisas feitas em trabalhos realizados no estado do Pará sobre pecuária bovina nos últimos 20 anos. Por último, as considerações finais.

\section{Revisão de literatura}

2.1. Pecuária bovina leiteira e agroecologia - um panorama

A agroecologia, enquanto campo do conhecimento ou até mesmo como paradigma de desenvolvimento (ALTIERI, 1989; VALENZUELA, 2016), teve grande evolução nas últimas duas décadas, tanto no meio acadêmico, quanto entre movimentos sociais vinculados às questões do campo e agricultura em geral (HERNANDES, 2020). No Brasil, embora avanços expressivos tenham sido observados, principalmente em sistemas de cultivo em relação à produção animal, temos visto avançar mais os trabalhos sobre aves e outros pequenos e médios animais (suínos, caprinos, ovinos), conforme diagnosticado no último Congresso Brasileiro de Agroecologia, em 2019.

Estudos sobre bovinocultura agroecológica têm sido muito mais no sentido da integração dos animais nos sistemas, principalmente para a ciclagem de nutrientes e favorecimento da adubação orgânica, ou em relação ao manejo sanitário, com foco em tratamentos homeopáticos. Ainda há um campo muito vasto para avançar, tanto em nível de reflexão sobre a construção, como em relação ao desenvolvimento de sistemas agroecológicos na produção animal. Dentre os fatores que estimulam a adoção de práticas agroecológicas, Couto e Navegantes (2016) indicam que há aqueles que são externos e os outros que são internos. Entre os externos, há destaque para o crédito, para a implantação de agroindústrias e para as capacitações; e as variáveis internas dizem respeito às decisões familiares, como o processamento dos produtos, a entrada de animais nos sistemas, entre outros. Os autores ponderam que a existência de grupos de camponeses que compartilham o interesse pela agroecologia aumenta as chances de que outros tomem a iniciativa de realizar a transição agroecológica.

São diversos os motivos apontados na literatura, para estimular a transição para agroecologia. Entre tais motivos, Magne et al. (2019) destacam que a autonomia dos povos e também dos territórios tanto é favorecida pela agroecologia em sistemas de 
produção animal, quanto traz pontos positivos em termos de desenvolvimento sustentável. Dale(2020) enfatiza a importância da agroecologia no cenário de mudanças climáticas e também no combate ao sistema agroalimentar predominante. Porém, baseado na abordagem gramsciana, há sugestão que a transição ocorra de modo justo, não priorizando apenas alguns nichos ou grupos. Além dessas indicações, há na literatura, estudos mostrando que a transição agroecológica pode trazer muitos ganhos econômicos, além da ampliação das redes de solidariedade, há também a manutenção da biodiversidade, entre outros resultados (OTSUKI; DE CASTRO, 2020).

De maneira mais pragmática, alguns trabalhos, indicavam práticas mais pontuais, no início do milênio, que poderiam ser consideradas agroecológicas, como a parcagem (ALVES; HOMMA; LOPES, 2005), o sistema caiçara e de retiros praticado por indígenas de Roraima (GONÇALVES; ALFAIA; DIAS-JUNIOR, 2019), que consistem em manejos que tornam viável a utilização do esterco animal para a adubação das áreas de cultivos. Em termos de alimentação e manejo das pastagens, há destaque para os trabalhos que apontam o Pastoreio Racional Voisin (PRV), a implantação de Banco de Proteínas (BP), pelo plantio de leguminosas forrageiras (Calapogônio Callopogonium mucunoides, Gliricidia - Gliricidia sepium e Alfafa - Medicago sativa L., entre outras), que servem de alimento, adubação e também como forma de sombreamento (BASTOS et al., 2018).

Para as questões de sanidade animal, baseada nos princípios agroecológicos, alguns estudos têm sido realizados para identificar plantas nativas em cada região que possuem potencial forrageiro ou medicamentoso (ARAÚJO, 2016). A fitoterapia e a homeopatia aparecem em destaque, seja para tratar doenças gástricas, ferimentos, parasitoses em geral (BASTOS et al., 2018; BALEM; MACHADO, 2019). Em relação ao bem-estar animal, estudo de Marques (2019) aponta que ainda pouco se avançou quanto ao reconhecimento e também às práticas que assegurem o conforto animal na criação de bovinos. O que mais se tem discutido é a importância do sombreamento, do espaço para circulação dos animais, controle de stress provocado pelo barulho e outras tensões. 


\section{Metodologia}

Este artigo faz uma reflexão a partir da experiência e estudos desenvolvidos por grupos de pesquisa atuantes em diversas equipes, incluindo os autores deste artigo, quem, ao longo de mais de 20 anos, têm estudado a pecuária leiteira na Amazônia paraense. Salientamos para o fato de que os estudos e resultados aqui utilizados são oriundos de: (i) trabalhos que não foram realizados com questionamento específico sobre agroecologia; (ii) uma diversidade de disciplinas, escalas, objetos e metodologias que foram analisados - cadeias, famílias, rebanhos, pastagens, práticas de manejos, experimentos de integração, entrevistas declarativas, medições em campo; (iii) de abrangência restrita em termos de sistema, com foco em áreas de colonização e nenhuma área de pecuária de várzea.

As pesquisas empíricas ocorreram nas regiões de terra firme da Transamazônica: Marabá, Redenção e São Felix do Xingu; e do nordeste paraense, nos municípios de Paragominas e Castanhal, resultando em publicações, como dissertações, teses, monografias, artigos e outros, como os trabalhos de: Ferreira (2001; 2003); Veiga et al. (2001); Machado (2001); Poccard-Chapuis et al (2003); Ludovino (2003); PoccardChapuis et al (2005); Houstiou (2006); Claudino (2007); Carvalho (2010); Carvalho (2012); Nogueira (2012); Navegantes et al. (2012); Malanski (2016); Maneschy et al. (2004); Maneschy et al. (2005a); Maneschy et al. (2005b).

Além disso, foi feita também uma pesquisa bibliográfica em busca de estudos contemporâneos que apresentassem o tema da agroecologia em sistemas leiteiros bovino.

Como grade de análise em nossa reflexão, iremos nos basear nos seguintes princípios da agroecologia: (i) uso e dependência de insumos externos; (ii) autonomia da família; (iii) segurança alimentar; (iv) diversificação do sistema; (v) conservação dos recursos naturais; (vi) bem-estar animal.

\section{Resultados e Discussão}

A pecuária leiteira na agricultura familiar do Pará é praticada principalmente pelos migrantes que vieram para região na década de 1970, inseridos ou não no programa oficial de colonização da Amazônia pela "pata do boi”, símbolo da efervescência e 
relevância da criação animal na constituição paraense.

Quanto às características dos rebanhos, pode-se dizer que a criação é de dupla finalidade, leite e corte. O leite tem a função primordial da renda mensal, permitindo às famílias gerenciarem as despesas cotidianas da casa e a manutenção do rebanho; enquanto a venda de bezerros, na fase de um ano ( $240 \mathrm{~kg}$ de peso vivo), serve para os investimentos mais importantes, tanto na propriedade como para a família. Este investimento corresponde desde a melhoria na infraestrutura da propriedade ao conforto da família, e reprodução social, como estudo dos filhos (NOGUEIRA, 2010; MALANSKI, 2012; FERREIRA et al., 2019).

Os efetivos do rebanho variam de tamanho por estabelecimento agrícola, podendo alcançar mais de 100 animais na região da transamazônica e em São Félix do Xingu em torno de 50-60 na região de Marabá (FERREIRA 2001; NOGUEIRA, 2010); e de 30-40 cabeças no município de Paragominas (COSTA, 2018). Em comum, tem-se o manejo alimentar exclusivamente a pasto, com fornecimento de sal mineral de forma irregular (tanto na composição quanto na frequência de fornecimento). As principais forrageiras utilizadas são: Brachiaria brizantha cv Marandu; Panicum maximum cv. Mombaça, Panicum. maximum cv. Massai, Brachiaria humidicola, e mais recentemente o novo cultivar de Panicum maximum, o BRS Zuri, conforme levantamento feito em pesquisa de campo realizada em 2019, nos municípios de Paragominas e Castanhal ${ }^{1}$.

O manejo é feito dividindo-se o rebanho em 2 lotes: (i) vacas em lactação e (ii) vacas secas, touro, bezerros desmamados. As áreas de pastos, nomeadas de piquetes ou mangas, são grandes, e a maioria (acima de 80\%) é utilizada sem o controle do tempo de pastejo/descanso. O que é controlado é a quantidade de biomassa produzida, privilegiando a entrada dos animais na área quando a forrageira já tem um desenvolvimento avançado, e retirando quando a mesma já está bem rasteira. Mesmo para aqueles que possuem mais de três áreas de pasto, o manejo rotacionado não é eficiente do ponto de vista da relação disponibilidade de capim versus quantidade de animais. Notase um desgaste progressivo no vigor da planta, que não é superado com técnicas de manejo da fertilidade dos solos e pastos, conforme registrado nos trabalhos de Carvalho (2010), Claudino (2011), Claudino et al. (2016).

\footnotetext{
${ }^{1}$ Projeto financiado pelo Banco da Amazônia, coordenado pela professora Soraya Abreu e pelo Núcleo de Agroecologia - Ajuri, coordenado pelo professor William Santos (no prelo).
} 
Em estudos realizados na microrregião de Castanhal e de Uruará, verificou-se que as pastagens formadas com Brachiaria brizantha cv Marandu não atenderam os requerimentos de $\mathrm{P}, \mathrm{Na}$ e $\mathrm{Cu}$ para bovinos de corte em fase de lactação (característica dos rebanhos de dupla aptidão), necessitando de suplementação via cocho (MANESCHY et al., 2005a; MANESCHY et al., 2005b). Em ambos os locais a estrutura e local dos cochos foi considerada inapropriada para o fornecimento de mistura mineral. Os relatos de apetite depravado, atraso no cio, fraturas espontâneas e o emagrecimento do rebanho sugerem deficiência de $\mathrm{P}$ e $\mathrm{Cu}$ sintetizando a baixa qualidade da nutrição dos animais (MANESCHY et al., 2004).

O manejo sanitário também não inclui grande intervenção de medicamentos alopáticos. São utilizados anti-inflamatórios e/ou antibióticos em casos mais extremos de mastite, e também aerossol contra larvas de mosca, sendo que este é utilizado inclusive no umbigo dos bezerros, a fim de secar o mesmo mais rapidamente. Em contrapartida, seguem o calendário de vacinação contra febre aftosa. Outros controles de zoonoses importantes, como a tuberculose e a brucelose, são realizados de forma esporádica.

$\mathrm{Na}$ contramão destas práticas extremamente extensivas, justificadas desde seu início pelo custo/benefício em investir pouco (principalmente pelo baixo preço da terra nos anos 1990-2000) e obter retorno, percebemos uma melhora nos rebanhos leiteiros. Enquanto na década de 90 e início dos anos 2000 podíamos considerar uma média de produtividade de 4,5 1 leite/vaca/dia como boa (MALANSKI, 2012), com animais chegando por vezes a atingir 7-8 1 leite/vaca/dia, atualmente tem-se animais produzindo ainda na marca dos 5 litros, ou bem abaixo de 21 leite/vaca/dia. Porém, uma parcela não negligenciável do rebanho (estimada em 10 a 30\%) alcançam produções acima de 101 leite/vaca/dia (SOUZA, 2019). Caracterizamos como um avanço, pois as condições precárias em que os rebanhos são criados, tanto em termos de manejo da alimentação (predominantemente a pasto de baixa qualidade), manejo sanitário ainda pouco desenvolvido, controle genético não acentuado, uma ordenha ao dia e poucas ações mais efetivas de bem-estar não favorecem ao aumento da produtividade. 


\subsection{Seria este um sistema agroecológico?}

Não há uma resposta direta entre ser ou não um sistema leiteiro agroecológico. Identificamos aspectos, por exemplo, de autonomia, relacionada à família em termos de decisão das práticas, da orientação da produção, da realização e distribuição do trabalho, assim como autonomia de insumos externos, que correspondem bem aos princípios agroecológicos.

Já o uso e gestão dos pastos implicam vários elementos que nos levam a refletir. Do ponto de vista da produção do pasto, a formação das pastagens sem os preparos e protocolos da produção mais intensiva, também se caracteriza como um sistema agroecológico. Fica mais difícil fazer esta análise quando o princípio norteador é a diversificação. Pensando pelo aspecto de que a pastagem visa à alimentação do rebanho, mantém a proteção do solo a partir da cobertura forrageira e se renova ao longo dos anos, também está dentro dos princípios expostos. Entretanto, a diversidade do uso do solo, quer seja intraparcela, quer seja em nível da propriedade, é baixa. No sistema a pasto, boa parte da área da propriedade é convertida no plantio de gramíneas. Neste aspecto, o sistema se distancia das práticas agroecológicas. Mas ao considerarmos o agroecossistema como um todo, há outras criações além de pequenas áreas com cultivos, o que leva a classificar o agroecossistema como diversificado.

Outras práticas frequentes na pastagem, que não se aproximam da agroecologia, são o uso do fogo anual para controle das plantas adventícias, e mais recentemente, o uso de herbicidas, também para o controle das adventícias. Todavia, durante a limpeza da pastagem é comum os agricultores familiares pouparem as espécies arbóreas adultas ou jovens com potencial para madeira, sombra, frutos ou forragem. Essas pastagens, arborizadas a partir do manejo da regeneração natural de espécies arbóreas, evidencia a biodiversidade funcional nesses agroecossistemas e aponta um caminho possível com baixo nível de insumos para a transição da monocultura de pastagens a sistemas agroflorestais pecuários - silvipastoris (MANESCHY et al., 2011a).

Nos aspectos sanitários, há também pontos que ora aproximam, ora distanciam dos princípios agroecológicos. Ao compararmos com sistemas intensivos, onde o uso de medicação e outros produtos para manter a higiene e a saúde dos animais são usados, nos 
estabelecimentos familiares, geralmente, não há prevenção e nem usos constantes, diários. Há uso sob necessidade. Os únicos medicamentos utilizados com regularidade anual são as vacinas. Ainda assim, somente a da campanha da aftosa é garantida. Aqui se tem um problema sanitário que afeta a saúde humana, pois doenças como brucelose, tuberculose não têm seu controle efetivo pelas instituições locais responsáveis.

Nos aspectos da soberania alimentar, a atividade funciona dentro dos princípios. As famílias têm a alimentação gerada pela produção leiteira e a venda e/ou troca, o que permite a obtenção de outros itens alimentícios. O uso dos resíduos, como o esterco e urina animal, são frequentes, coadunando com as propostas da agroecologia. Porém, a quantidade produzida é ainda muito aquém da necessária para auxiliar na fertilidade e nos processos de recomposição da matéria orgânica do solo.

De uma forma ampla, a pecuária leiteira descrita aqui pode ser classificada como um sistema natural e sem muito incrementos, fruto da percepção e experiências empíricas dos agricultores e pecuaristas familiares sobre as necessidades de interação visando a produção dentro do novo ecossistema. Ao longo dos anos, a capacidade dos recursos naturais em se manterem, mesmo em condições limitantes, fez com que permanecessem e pudessem se reproduzir com estas práticas até os tempos atuais. Mas os mesmos não estão estáticos, sendo constatadas mudanças nos últimos anos (POCCARD-CHAPUIS et $a l ., 2015)$, em busca de novos ganhos produtivos, havendo a previsão de que outros caminhos e alternativas devem ser apresentados.

A produção intensiva tem seus protocolos definidos. A agroecologia nem tanto, esta tem princípios norteadores para construção de práticas coerentes com o ecossistema. Retornamos assim à questão inicial: o que seria um sistema leiteiro agroecológico nas condições da Amazônia paraense?

4.2. Limites e potencialidades para sistemas de produção leiteira agroecológicos: quais as perspectivas?

Apesar de, até o momento a atividade ser conduzida sem uma gestão equilibrada entre produção, produtividade e recursos naturais disponíveis, o sistema praticado pelas famílias, aliado às condições ambientais da região, favorecem mudanças na direção de composição de práticas de criação agroecológicas. O primeiro ponto de destaque é que o 
manejo geral desenvolvido até o momento não tem dependência dos insumos químicos. Aliado a este fato temos o clima, que é favorável a uma produção de biomassa, possibilitando formas de reorganização no manejo do pastejo. Prosseguindo nesta mesma direção, há uma diversificação das forrageiras utilizadas, através da introdução de leguminosas arbóreas, arbustivas e/ou rasteiras, como formas de promover melhor condicionamento climático aos animais, melhor nutrição dos mesmos, assim como proteção quanto a desidratação das gramíneas e melhoria na fertilidade do solo.

A arborização das áreas de pastagens, via manejo da regeneração natural, pode conferir maior biodiversidade funcional e resiliência aos agroecossistemas pecuários (SANTOS; MITJA, 2011). Agricultores familiares e pecuaristas compreendem a importância dessa associação como benéfica, mas citam dificuldades técnicas e de fomento para estabelecer esses sistemas em suas propriedades (VEIGA et al., 2006; MANESCHY, 2009; MANESCHY et al., 2011b)

Quanto ao manejo genético, alguns passos já foram dados, com introdução de raças com perfil adaptado às condições regionais e de boa produtividade nos últimos anos, ampliando as chances de realização de seleção massal dentro do rebanho. Juntos, esses fatores possibilitam avanços importantes ao equilíbrio ganho produtivo e para a conservação dos agroecossistemas.

Como principais limitantes aos processos de mudanças, destacamos a falta de formalização e divulgação de alternativas testadas que possam ser utilizadas, assim como a capacitação dos agricultores na nova forma de gerir o recurso natural que é a pastagem. Também se faz necessário investir nas práticas de manejo da sanidade animal, conforme destacado por Balem e Machado (2019), que incluam fitoterapia e homeopatia, além de adoção de práticas que favoreçam o bem-estar animal.

\section{Conclusões}

O texto apresentou uma síntese de pesquisas realizadas ao longo das últimas duas décadas, em diferentes regiões do Estado do Pará, sobre a pecuária bovina de leite em estabelecimentos familiares. Primeiramente, foi feita uma caracterização geral dos sistemas de produção leiteira, seguida de apresentação dos princípios gerais preconizados como agroecológicos. O perfil de sistemas produtivos permitiu cotejar a situação regional 
com os dados da literatura teórica sobre práticas agroecológicas.

Diante do exposto, concluímos que não há uma classificação exata para o sistema leiteiro de base familiar na Amazônia paraense, podendo-se dizer apenas que se trata, de uma maneira geral, uma classificação de sistemas naturais e sem muitos incrementos, fruto da percepção e experiências empíricas dos atores sobre as necessidades de interação visando à produção dentro do novo ecossistema. Apesar de coexistir há mais de 40 anos na região, as mudanças são lentas e não atingiu o patamar de produção intensiva para falarmos de uma transição para sistema agroecológico. É um sistema antigo, porém "bruto", no sentido de não ser elaborado, mas de se caracterizar como uma ação em face à adversidade, que avança na medida em que encontra uma barreira.

O incremento, no povoamento forrageiro com espécies de alta qualidade nutricional com leguminosas que possam suprir adequadamente os requerimentos animais diminuindo a necessidade de insumos externos a propriedade aliado ao controle do pastoreio e manejo da regeneração natural do componente arbóreo, são caminhos possíveis para todos os sistemas existentes.

É necessário leituras como a proposta neste artigo, a fim de aprofundar os aspectos agroecológicos desta produção e, dentro dos diferentes contextos amazônicos, apontar caminhos para o desenvolvimento de sistemas agroecológicos compatíveis com a cultura e o ecossistema local. 


\section{Referências}

ALTIERI, Miguel. Agroecology: a new research and development paradigm for world agriculture. Agriculture, Ecosystems \& Environment, v. 27, n. 1-4, p. 37-46, 1989.

ALTIERI, M. Colheita fatal: velhas e novas dimensões da tragédia ecológica da agricultura moderna. IN: Agroecologia: bases científicas para uma agricultura sustentável. $3^{\text {a }}$ Ed. Revista e Ampliada, Miguel Altieri, Ed. Expressão Popular, São Paulo, 2012.

ALVES, Raimundo Nonato Brabo; HOMMA, Alfredo Kingo Oyama; LOPES, Otávio Manoel Nunes. $O$ método de parcagem como alternativa agroecológica para a integração agricultura/pecuária da produção familiar do sudeste paraense. Belém: Embrapa Amazônia Oriental, 2005.

ARAÚJO, Paula de Carvalho Machado. Identificação e avaliação de espécies de interesse forrageiro e a percepção de produtores familiares da Reserva de Desenvolvimento Sustentável Amanã, Amazonas, Brasil. 2016. 93f. Dissertação (Mestrado Profissional em Agricultura Orgânica) - Universidade Federal Rural do rio de Janeiro, Seropédica, 2016.

BALEM, Tatiana Aparecida; MACHADO, Ricardo Lopes. Sistemas de produção de leite de base ecológica: a construção das variáveis a partir de uma experiência de extensão rural em Santa Maria (RS). Rev. Brasileira de Agroecologia. v. 14, n.1. 2019.

BASTOS, Sintia Barboza et al. Transição agroecológica da pecuária em Cunha - SP e região. Cadernos de Agroecologia, v. 13, n. 1, 2018.

BORBA, Marcos; TRINDADE, José Pedro. Desafios para conservação e a valorização da pecuária sustentável. In: PILLAR, Valério de Patta et al. (Ed.). Campos sulinos: conservação e uso sustentável da biodiversidade. Brasília, DF: MMA, 2009.

CAPORAL, Francisco Roberto. Extensão Rural e Agroecologia: temas sobre um novo desenvolvimento rural, necessário e possível. Brasília: MDA, 2007

CARVALHO, Soraya Abreu de; TOURRAND, Jean-François; POCCARD-CHAPUIS, René. Atividade leiteira: um desafio para a consolidação da agricultura familiar na região da Transamazônica, no Pará. Cadernos de ciência e tecnologia, v.29, 2012.

CARVALHO, Adson Jonnas. A busca da sustentabilidade das pastagens no Assentamento Belo Horizonte I, São Domingos do Araguaia - PA. Dissertação (Mestrado em Agriculturas Familiares e Desenvolvimento Sustentável) - Universidade Federal do Pará, Belém, 2010.

COUTO, Xoán Carlos Sánchez; ALVES, Lívia de Freitas Navegantes. Aspectos históricos relacionados à adoção de práticas agroecológicas: análise retrospectiva no 
Oeste maranhense. Novos Cadernos NAEA, v. 19, n. 1, 2016.

CLAUDINO, Livio Sérgio Dias. Estudo das mudanças no manejo alimentar e melhoramento genético dos bovinos leiteiros da região de Marabá: o caso de Murumuru - PA. 2007. Monografia (Graduação em Agronomia) - Universidade Federal do Pará, Marabá. 2007.

CLAUDINO, Livio Sérgio Dias. Ocupação dos espaços, gestão e degradação das pastagens entre pecuaristas da microrregião de São Félix do Xingu. Dissertação (Mestrado em Agriculturas Familiares e Desenvolvimento Sustentável) - Universidade Federal do Pará, Belém, 2011.

CLAUDINO, Livio Sérgio Dias; FERREIRA, Laura Angélica; POCCARD-CHAPUIS, René. A diversidade de condições socioeconômicas dos pecuaristas e a gestão das pastagens no Sul do Pará. Rev. Bras. Gestão e Des. Reg. v. 12, 2016.

DALE, Bryan. Alliances for agroecology: from climate change to food system change. Agroecology and Sustainable Food Systems, v. 44, n. 5, p. 629-652, 2020.

FERREIRA, Laura Angelica. Evolução e Perspectivas para a Agricultura Familiar do Município de Uruará: pistas para uma reflexão sobre a consolidação dos sistemas de produção agrícolas familiares. In: SIMÕES, A. (Org.). Coleta Amazônica: iniciativas em pesquisa, formação e apoio ao desenvolvimento rural sustentável na Amazônia. Belém: Alves, 2003.

GONÇALVES, Ludmilla Verona Carvalho; ALFAIA, Sonia Sena; DIAS-JUNIOR, Carlos Machado. O Manejo das Caiçaras Indígenas: Uma prática agropecuária no Lavrado de Roraima, Amazônia brasileira. Mundo Amazónico, v. 10, n. 1, 2019.

HERNANDEZ, Andreas. The emergence of agroecology as a political tool in the Brazilian Landless Movement. Local Environment, v. 25, n. 3, p. 205-227, 2020.

LUDOVINO, Rui. Evolução e viabilidade dos sistemas de agricultura familiar na Região bragantina - Pará - Brasil. In: TOURRAND, Jean-François; VEIGA, Jonas Bastos da (Orgs.). Viabilidade dos Sistemas Agropecuários na Agricultura Familiar da Amazônia. Belém: Embrapa Amazônia Oriental, 2003. 486p.

MAGNE, Marie-Angélina et al. An Integrated Approach to Livestock Farming Systems' Autonomy to Design and Manage Agroecological Transition at the Farm and Territorial Levels. In: Agroecological Transitions: From Theory to Practice in Local Participatory Design. Springer, Cham, 2019. p. 45-68.

MALANSKI, Priscila Duarte. Introdução de leguminosas forrageiras em sistemas de criação leiteiros no assentamento Belo Horizonte I, São Domingos do Araguaia PA. 91f. Dissertação (Mestrado em Agriculturas Familiares e Desenvolvimento Sustentável) - Universidade Federal do Pará, Belém, 2012.

MANESCHY, Rosana Quaresma; CARDOSO, Elyzabeth Cruz; VEIGA, Jonas Bastos; MCDOWELL, Lee. R. Effect of season on mineral concentration of Braquiarão 
(Brachiaria brizantha cv. Marandu) dairy cattle production in the Eastern Amazon. I. Macrominerals. Journal of Animal and Veterinary Advances, v.4, p.123 - 126, 2005a.

MANESCHY, Rosana Quaresma; CARDOSO, Elyzabeth Cruz; VEIGA, Jonas Bastos; MCDOWELL, Lee. Effect of season on mineral concentration of Braquiarão (Brachiaria brizantha cv. Marandu) dairy cattle production in the Eastern Amazon. II. Microminerals. Journal of Animal and Veterinary Advances, v.4, p.118 - 122, $2005 b$.

MANESCHY, Rosana Quaresma; CARDOSO, Elizabeth Cruz; VEIGA, Jonas Bastos. Características da suplementação leiteira na Amazônia Oriental, Microrregião de Castanhal e município de Uruará. Movendo Ideias (UNAMA), v.9, p.70 - 78, 2004.

MANESCHY, Rosana Quaresma et al. Diálogo de saberes e processo de construção de inovação agroflorestal em estabelecimentos rurais familiares no sudeste do Pará In: Práticas Agroecológicas: Soluções sustentáveis para a agricultura familiar na região sudeste do Pará ed. Jundiaí: Paco Editorial, 2011b, p. 237-255.

MANESCHY, Rosana Quaresma et al. Manejo da regeneração natural de espécies arbóreas na pastagem como alternativa silvipastoril para a sustentabilidade da agricultura familiar no sudeste do Pará In: Práticas Agroecológicas: Soluções sustentáveis para a agricultura familiar na região sudeste do Pará ed. Jundiaí: Paco Editorial, 2011a, p. 289-306.

MARQUES, Viviane Osmarina de Almeida. Estudos preliminares sobre a relevância do tema bem-estar animal na tomada de decisão dos pecuaristas no estado do Pará. 40 f. Trabalho de Conclusão de Curso (Graduação em Agronomia) Universidade Federal Rural da Amazônia, Campus Belém, 2019.

NAVEGANTES-ALVES, Lívia et al. Grassland deterioration linked to farm trajectories in the eastern Amazon. Outlook on Agriculture, v. 41, p. 195-201, 2012.

NOGUEIRA, Simone Silva. Intensificação ou diversificação? A pecuária leiteira em questão. 2012. 153f. Dissertação (Mestrado em Agriculturas Familiares e Desenvolvimento Sustentável) - Universidade Federal do Belém, Belém, 2012.

OTSUKI, Kei; DE CASTRO, Fabio. Solidarity Economy in Brazil: Towards Institutionalization of Sharing and Agroecological Practices. In: SAITO, Osamu (org.). Sharing Ecosystem Services: Building More Sustainable and Resilient Society. Springer, Singapore, 2020. p. 159-178.

POCCARD-CHAPUIS, Rene et al. A cadeia produtiva do leite: uma alternativa para consolidar a agricultura familiar nas frentes pioneiras da Amazônia? In: TOURRAND, Jean-François; VEIGA, Jonas Bastos da. Viabilidade de sistemas agropecuários na agricultura familiar da Amazônia. Belém: Embrapa Amazônia Oriental, 2003.

SANTOS, Aléssio Moreira dos; MITJA, Dani. Pastagens arborizadas no projeto de assentamento Benfica, município de Itupiranga, Pará, Brasil. Rev. Árvore [online]. 
2011, v. 35, n. 4, p. 919-930.

SILVA, Edfranklin Moreira da, NAVEGANTES-ALVES, Livia. A ocupação do espaço pela dendeicultura e seus efeitos na produção agrícola familiar na Amazônia Oriental.

Cofins - Revue franco-brésilienne de géographie. n. 30, 2017.

SOUZA, Jorge Oliveira. O uso do controle leiteiro como ferramenta de análise da produção leiteira familiar: O caso da Comunidade Nova Jerusalém em

Paragominas - PA. 2019. 25f. Trabalho de Conclusão de Curso (Graduação em Medicina Veterinária) - Universidade Federal do Pará, Castanhal, 2019.

VALENZUELA, Hector. Agroecology: a global paradigm to challenge mainstream industrial agriculture. Horticulturae, v. 2, n. 1, p. 2, 2016.

VEIGA, Jonas Bastos et al. Produção leiteira e o desenvolvimento regional na Amazônia Oriental. Belém: Embrapa Amazônia Oriental, 2001. 24p.

VEIGA, Jonas Bastos; MANESCHY, Rosana Quaresma; DUTRA, S. Potencial de adoção de sistemas silvipastoris por produtores da região Transamazônica. In: Sistemas agroflorestais e desenvolvimento com proteção ambiental: Práticas e tecnologias desenvolvidas.1 ed. Colombo: Embrapa Florestas, 2006, p. 95-106.

MONTOYA, Luciano Javier; RIBASKI, Jorge; MACHADO, Antonio Maciel Botelho. Sistemas agroflorestais e desenvolvimento com proteção ambiental: Práticas e tecnologias desenvolvidas. Colombo, Brasil: Embrapa Florestas, 2006. p. 95-106. 